\title{
Multi-azimuthal-angle effects in self-induced supernova neutrino flavor conversions without axial symmetry
}

\author{
Alessandro Mirizzi ${ }^{1}$ \\ ${ }^{1}$ II Institut für Theoretische Physik, Universität Hamburg, \\ Luruper Chaussee 149, 22761 Hamburg, Germany
}

\begin{abstract}
The flavor evolution of neutrinos emitted by a supernova (SN) core is strongly affected by the refractive effects associated with the neutrino-neutrino interactions in the deepest stellar regions. Till now, all numerical studies have assumed the axial symmetry for the "multi-angle effects" associated with the neutrino-neutrino interactions. Recently, it has been pointed out in [1] that removing this assumption, a new multi-azimuthal-angle (MAA) instability would emerge in the flavor evolution of the dense SN neutrino gas in addition to the one caused by multi-zenith-angle (MZA) effects. Inspired by this result, for the first time we numerically solve the non-linear neutrino propagation equations in SN, introducing the azimuthal angle as angular variable in addition to the usual zenith angle. We consider simple energy spectra with an excess of $\nu_{e}$ over $\bar{\nu}_{e}$. We find that even starting with a complete axial symmetric neutrino emission, the MAA effects would lead to significant flavor conversions in normal mass hierarchy, in cases otherwise stable under the only MZA effects. The final outcome of the flavor conversions, triggered by the MAA instability, depends on the initial asymmetry between $\nu_{e}$ and $\bar{\nu}_{e}$ spectra. If it is sufficiently large, final spectra would show an ordered behavior with spectral swaps and splits. Conversely, for small flavor asymmetries flavor decoherence among angular modes develops, affecting the flavor evolution also in inverted mass hierarchy.
\end{abstract}

PACS numbers: 14.60.Pq, 97.60.Bw

\section{INTRODUCTION}

Flavor conversions of supernova neutrinos are a fascinating problem involving refractive effects that not only depend on the ordinary matter background [2, 3], but also on the neutrino fluxes themselves. Indeed, neutrinoneutrino interactions provide a non-linear term in the equations of motion [4 6] that causes collective flavor conversions $7-14$. Since few years $15-17$ it has realized that in the SN context these self-induced effects give rise to qualitatively new phenomena (see, e.g., [18] for a recent review). The main consequence of this unusual type of flavor transitions is an exchange of the spectrum of the electron species $\nu_{e}\left(\bar{\nu}_{e}\right)$ with the non-electron ones $\nu_{x}$ $\left(\bar{\nu}_{x}\right)$ in certain energy intervals, giving rise to interesting spectral features [16 28, 30, 47]. These flavor exchanges are called "swaps" marked by the "splits", which are the boundary features at the edges of each swap interval.

In this context, one of the main complication in the simulation of the flavor evolution is that the flux of neutrinos emitted from a supernova core is far from isotropic. The current-current nature of the weakinteraction Hamiltonian implies that the interaction energy between neutrinos of momenta $\mathbf{p}$ and $\mathbf{q}$ is proportional to $\left(1-\mathbf{v}_{\mathbf{p}} \cdot \mathbf{v}_{\mathbf{q}}\right)$, where $\mathbf{v}_{\mathbf{p}}$ is the neutrino velocity [7, 31]. In a non-isotropic medium this velocitydependent term would not average to zero, producing a different refractive index for neutrinos propagating on different trajectories. This is the origin of the so-called "multi-angle effects" 16], which hinder the maintenance of the coherent oscillation behavior for different neutrino modes [16, 19, 32 34]. All the numerical simulations of supernova neutrino flavor conversions have characterized the multi-angle effects in the so called "bulb model" [16] in which neutrinos are assumed spherically emitted at the neutrino-sphere at a radius $r=R .{ }^{1}$ Global spherical symmetry was also assumed in the neutrino propagation, reducing to an axial symmetry of the multi-angle effects along the radial line-of-sight (polar axis). Therefore, at any radius $r>R$ along the polar axis, neutrinos with different momenta $\mathbf{p}$ (characterized by $|\mathbf{p}|=E$ ) were identified by the only zenith angle at the emission $\vartheta_{R}$.

The underlying assumption in the bulb model is that small deviations with respect to a perfect axial symmetry, would not significantly affect the neutrino flavor evolution. This unjustified assumption has been questioned in a recent paper [1], in which the authors investigated the effect of relaxing the axial symmetry, performing a linearized stability analysis of the SN neutrino equations of motion, introducing the azimuthal angle of neutrino propagation $\varphi$ as explicit variable. The stability analysis, as described in [37], would allow one to determine the possible onset of the flavor conversions, seeking for an exponentially growing solution of the eigenvalue problem, associated with the linearized equations of motion for the neutrino ensemble. Surprisingly it has been found that even assuming a completely spherically symmetric neutrino emission at the neutrinosphere, without forcing the neutrino ensemble to follow the axial symmetric solution, a new "multi-azimuthal-angle" (MAA) instability would emerge in addition to the one caused by the "multizenith-angle" (MZA) effects. In particular, considering simple neutrino ensembles with only $\nu_{e}$ and $\bar{\nu}_{e}$, the in-

\footnotetext{
1 The effect of non-trivial $\nu$ zenith angle distributions at the neutrinosphere has been discussed in [35, 36].
} 
stability has been found in normal mass hierarchy (NH, $\Delta m_{\text {atm }}^{2}=m_{3}^{2}-m_{1,2}^{2}>0$ ), where the system would have been stable imposing the perfect axial symmetry. Subsequently, the role of these instability has been clarified in [38], where it has been shown with simple toy models that, introducing small deviations in the assumed symmetries of the initial conditions in a dense $\nu$ gas, new instabilities would be triggered.

Once this new instability has been found in the dense SN neutrino gas, numerical simulations of the flavor evolution are mandatory in order to characterize how the instable system evolves. At this regard, the main aim of this work is to perform a numerical study of the SN neutrino flavor evolution including both neutrino zenith and azimuthal propagation angles. It it known that the development of self-induced flavor conversions in SNe, as well as their dependence on the neutrino mass hierarchy, is crucially dependent on the flux ordering among different neutrino species. Since our primary goal is to have a first characterization of possible effects induced by the MAA instability, we will consider simple neutrino systems, whose flavor evolution is well understood in the axial symmetric case. We leave the study of more complicated spectral configurations to future works.

The structure of the paper is as follows. In Sec. 2 we present the neutrino equations of motion without imposing axial asymmetry. In Sec. 3 we show the results for the flavor evolution of a monochromatic beam of $\nu_{e}$ and $\bar{\nu}_{e}$ with different initial flavor asymmetries, in the presence of only MAA and both MAA and MZA effects. We find that independently of the initial flavor symmetry, the MAA effects trigger new flavor conversions in the (otherwise stable) NH case. These lead to a flavor decoherence of the neutrino ensemble. In Sec. 3 we also include the effect of continuous energy spectra. We consider a system with an excess of $\nu_{e}$ over $\bar{\nu}_{e}$, whose spectra are represented by Fermi-Dirac distributions. Again we find new flavor conversions in $\mathrm{NH}$ case. Remarkably, the flavor evolution crucially depends on the asymmetry among $\nu_{e}$ and $\bar{\nu}_{e}$. If it is sufficiently large, final spectra show an ordered behavior with spectral swaps and splits. Conversely, for small flavor asymmetries decoherence among angular modes develops, affecting the flavor evolution also in inverted mass hierarchy (IH, $\left.\Delta m_{\mathrm{atm}}^{2}<0\right)$. Finally, in Sec. 4 we summarize our results and we conclude.

\section{EQUATIONS OF MOTION}

We work in a two-flavor oscillation scenario, associated to the atmospheric mass-square difference $\Delta m_{\text {atm }}^{2}=$ $2 \times 10^{-3} \mathrm{eV}^{2}$ and and with the small (matter suppressed) in-medium mixing $\Theta=10^{-3}$. Since we aim at isolating the effect of the MAA instability, we assume in the following that self-induced flavor conversions are not matter suppressed (as expected instead at $t \lesssim 1 \mathrm{~s}$ after the core bounce) 39 42, ignoring the ordinary matter back- ground in the equations of motion.

We still assume that variations in the transverse direction are small so that the flavor evolution depends only on $r, E$ and $\mathbf{v}_{\mathbf{p}}$. In other words, we study neutrino propagation only in the neighborhood of a chosen location and do not worry about the global solution [38]. We assume neutrinos to be emitted half-isotropically at the neutrinosphere, that here we schematically fix at $R=10 \mathrm{~km}$. For simplicity, we neglect possible residual scatterings that could affect $\nu$ 's after the neutrinosphere, producing a small "neutrino halo" that would broaden the $\nu$ angular distributions [43, 44]. If we describe neutrinos by their local velocity $\mathbf{v}_{\mathbf{p}}$, the zenith angle of a given neutrino trajectory with respect to the radial direction $\theta_{r}$, due to flux conservation will depend on the radial coordinate $r$ even for straight line propagation. It is thus convenient to parameterize every zenith angular mode in terms of its emission angle $\theta_{R}$ relative to the radial direction of the neutrinosphere. A further simplification is obtained if one labels the different zenith angular modes in terms of the variable $u=\sin ^{2} \theta_{R}$, as in [33, 37]. At a radius $r$, the radial velocity of a mode with angular label $u$ is $v_{u, r}=\left(1-u R^{2} / r^{2}\right)^{1 / 2}$ [33] and the transverse velocity is $\beta_{u, r}=u^{1 / 2} R / r$ [1]. Conventionally, we use negative $E$ for anti-neutrinos and we label the different energy modes by the vacuum oscillation frequency $\omega=\Delta m_{\text {atm }}^{2} / 2 E$. Then, following [33], we define the flux matrices $\mathbf{J}_{\omega, u, \varphi}$ as function of the radial coordinate, where $\varphi$ is the azimuth angle of $\mathbf{v}_{\mathbf{p}}$. The diagonal $\mathbf{J}_{\omega, u, \varphi}$ elements are the ordinary number fluxes $F_{\nu_{\alpha}}(\omega, u, \varphi)$ at a radius $r$. The off-diagonal elements, which are initially zero, carry a phase information due to flavor mixing. The flux matrices are represented by polarization vectors $\mathbf{P}_{\omega, u, \varphi}$ in the usual way,

$$
\mathbf{J}_{\omega, u, \varphi}=\frac{\operatorname{Tr} \mathbf{J}_{\omega, u, \varphi}}{2}+\frac{F_{e}^{R}-F_{x}^{R}}{2} \mathbf{P}_{\omega, u, \varphi} \cdot \boldsymbol{\sigma}
$$

where $F_{e, x}^{R}(\omega, u, \varphi)$ are the flavor fluxes at the neutrinosphere radius $R$. Following [1], we introduce the dimensionless spectrum $g(\omega, u, \varphi)$, representing $F_{e}^{R}-F_{x}^{R}$. It is negative for antineutrinos, where $\omega<0$, and normalized to $\bar{\nu}$ flux, i.e. $\int_{-\infty}^{0} d \omega \int_{0}^{1} d u \int_{0}^{2 \pi} d \varphi g(\omega, u, \varphi)=-1$. We introduce the $\nu-\bar{\nu}$ asymmetry parameter $\varepsilon=\int d \Gamma g$, where $\int d \Gamma=\int_{-\infty}^{+\infty} d \omega \int_{0}^{1} d u \int_{0}^{2 \pi} d \varphi$. In the following we will always assume axial symmetry of the neutrino emission and half-isotropic zenith angle $\nu$ distributions. Therefore, at the neutrinosphere $g(\omega, u, \varphi)=g(\omega) / 2 \pi$.

We write the compact form of the the equations of motion (neglecting matter effects) for the flavor-spin polarization vectors $\left[1,\left[\underline{5},[37]^{2}\right.\right.$

\footnotetext{
${ }^{2}$ In the flavor-spin polarization vector formalism, $\nu$ and $\bar{\nu}$ of the same flavor have polarization vectors which point in opposite directions [18]. This formalism allows to write in a compact from the equations of motion for neutrinos and antineutrinos
} 


$$
\begin{aligned}
\mathrm{i} \partial_{r} \mathbf{P}_{\omega, u, \varphi} & =\frac{\omega \mathbf{B} \times \mathbf{P}_{\omega, u, \varphi}}{v_{u}} \\
& +\mu_{R} \frac{R^{2}}{r^{2}} \int d \Gamma^{\prime}\left(\frac{1-v_{u} v_{u^{\prime}}-\boldsymbol{\beta} \cdot \boldsymbol{\beta}^{\prime}}{v_{u} v_{u^{\prime}}}\right) \\
& \times g\left(\omega^{\prime}\right) \mathbf{P}_{\omega^{\prime}, u^{\prime}, \varphi^{\prime}} \times \mathbf{P}_{\omega, u, \varphi},
\end{aligned}
$$

where $\mathbf{B}=(\sin 2 \Theta, 0, \pm \cos 2 \Theta)$, with $B_{z}<0$ corresponds to NH, while $B_{z}>0$ to IH. The strength of the $\nu-\nu$ interaction is parametrized as

$$
\mu_{R}=\sqrt{2} G_{F} \frac{\Phi_{\bar{\nu}_{e}}(R)-\Phi_{\bar{\nu}_{x}}(R)}{4 \pi R^{2}},
$$

normalized to the total $\bar{\nu}_{e}-\bar{\nu}_{x}$ flux difference at $R$. Finally, $\boldsymbol{\beta} \cdot \boldsymbol{\beta}^{\prime}=\sqrt{u u^{\prime}} R^{2} / r^{2} \cos \left(\varphi-\varphi^{\prime}\right)$. This term breaks the axial symmetry.

We expect that self-induced flavor conversions in $\mathrm{SNe}$ would develop at $r \gg R$. Therefore, we can adopt the large-distance approximation of Eq. (2) getting [1]

$$
\begin{aligned}
\mathrm{i} \partial_{r} \mathbf{P}_{\omega, u, \varphi} & =\omega \mathbf{B} \times \mathbf{P}_{\omega, u, \varphi} \\
& -\mu \int d \Gamma^{\prime}\left[u+u^{\prime}-2 \sqrt{u u^{\prime}} \cos \left(\varphi-\varphi^{\prime}\right)\right] \\
& \times g\left(\omega^{\prime}\right) \mathbf{P}_{\omega^{\prime}, u^{\prime}, \varphi^{\prime}} \times \mathbf{P}_{\omega, u, \varphi},
\end{aligned}
$$

where

$$
\mu=\mu_{R} \frac{R^{4}}{2 r^{4}} .
$$

In order to numerically solve Eq. (44) we use an integration routine for stiff ordinary differential equations taken from the NAG libraries [45] and based on an adaptive method.

\section{SINGLE ENERGY NEUTRINO BEAM}

In order to perform a first numerical exploration of the solution of Eq. (4) in the presence of the MAA effects, we consider a monochromatic $\nu$ ensemble, composed only by $\nu_{e}$ and $\bar{\nu}_{e}$ in which all the particles share the same energy $\langle E\rangle=15 \mathrm{MeV}$. This would correspond to a vacuum oscillation frequency $\omega_{0}=0.34 \mathrm{~km}^{-1}$. We consider an excess of $\nu_{e}$ over $\bar{\nu}_{e}$, parametrized by the asymmetry parameter $\varepsilon$, such that the initial energy spectrum would be equivalent to $g(\omega)=-\delta\left(\omega+\omega_{0}\right)+(1+\varepsilon) \delta\left(\omega-\omega_{0}\right)$. We fix the $\bar{\nu}_{e}$ luminosity $L_{\bar{\nu}_{e}}=10^{51} \mathrm{erg} / \mathrm{s}$, so that in Eq. (3)

$$
\begin{aligned}
\mu_{R} & =0.7 \times 10^{5} \mathrm{~km}^{-1} \\
& \times \frac{L_{\bar{\nu}_{e}}}{\langle E\rangle} \frac{15 \mathrm{MeV}}{10^{51} \mathrm{erg} / \mathrm{s}}\left(\frac{10 \mathrm{~km}}{R}\right)^{2} .
\end{aligned}
$$

[see Eq. 22] . However, in our numerical examples we prefer to use the convention in which the polarization vectors for $\nu$ and $\bar{\nu}$ point in the same direction.
The flavor evolution in this case under perfect axial symmetry is well known. If the flavor asymmetry $\varepsilon$ is sufficiently large (i.e. $\varepsilon \gtrsim 0.3$ ) the system would be stable in normal hierarchy, while in inverted hierarchy it would exhibit the bimodal instability leading to complete flavor conversions in the $\bar{\nu}$ sector, while in the $\nu$ sector there will remain a fraction of $\nu_{e}$ fixed by the conservation of the initial lepton asymmetry $\varepsilon$ 17]. Conversely for smaller values of $\varepsilon$, MZA effects would lead to flavor decoherence in both the mass hierarchies [33].

We show how the flavor evolution changes including the MAA effects. In Fig. 1 we represent the radial evolution of $z$-component $\bar{P}_{z}$ of the $\bar{\nu}$ polarization vector integrated over $\varphi$ and $u$. In the Figure, we normalize the integrated polarization vector such as $\bar{P}_{z}=+1$ corresponds to a pure $\bar{\nu}_{e}$ system, while $\bar{P}_{z}=-1$ corresponds to all $\bar{\nu}_{x}$. We consider both NH (left panels) and IH (righ panels) for different values of the flavor asymmetry parameter, namely $\varepsilon=1.5$ (upper panels), $\varepsilon=0.5$ (middle panels) and $\varepsilon=0.3$ (bottom panels). We compare single-zenithangle (SZA) evolution (dashed curves) with $u=0.5$, with the complete MZA and MAA cases (continuous curves). We realize that in order to get numerical convergence of our results is enough to take $\mathcal{O}(10)$ angular modes in $\varphi$, while a larger number of modes is required for the MZA simulations. We remark that numerically stable results require less modes in $\varphi$ than in $u$. Indeed, as discussed in [1] for discrete azimuthal angles no spurious instabilities appear, in contrast to discrete zenith angles [46]. In particular, we use $N_{\varphi}=30$ for $\varphi \in[0 ; 2 \pi]$ and $N_{u}=80$ for $u \in[0 ; 1]$.

The most striking result of this toy model is that independently of the value of the flavor asymmetry $\varepsilon$, if we break the axial symmetry in the $\nu$ evolution, $\mathrm{NH}$ presents self-induced flavor conversions, as predicted from the stability analysis of [1]. It is interesting to mention that these flavor conversions develop even if here we are starting from an initial perfectly spherical $\nu$ distribution. Indeed, since the system in unstable it is enough a small breaking of the perfect axial symmetry, induced the numerical noise: The small numerical seeds will be exponentially amplified during the flavor evolution. ${ }^{3}$ The effect of $\varepsilon$ is to shift the onset of the flavor conversions, i.e. the largest $\varepsilon$ the greatest the onset radius of the flavor conversions. This relation is qualitatively similar to what already found for the bimodal flavor evolution [17. It is also interesting to notice that MZA effects do not seem to play an important role in changing the flavor dynamics. The final outcome is generic: the MAA effect leads to a final $\bar{P}_{z} \simeq 0$ that would imply a flavor decoherence leading to a mixture of $\bar{\nu}_{e}$ and $\bar{\nu}_{x}$. Of course, the decoherence cannot be complete in the $\nu$ sector due to the lepton number conservation [17], that guarantees

\footnotetext{
3 The speed-up of flavor instabilities under the effects of very small seeds in the initial conditions was already pointed out in [47].
} 

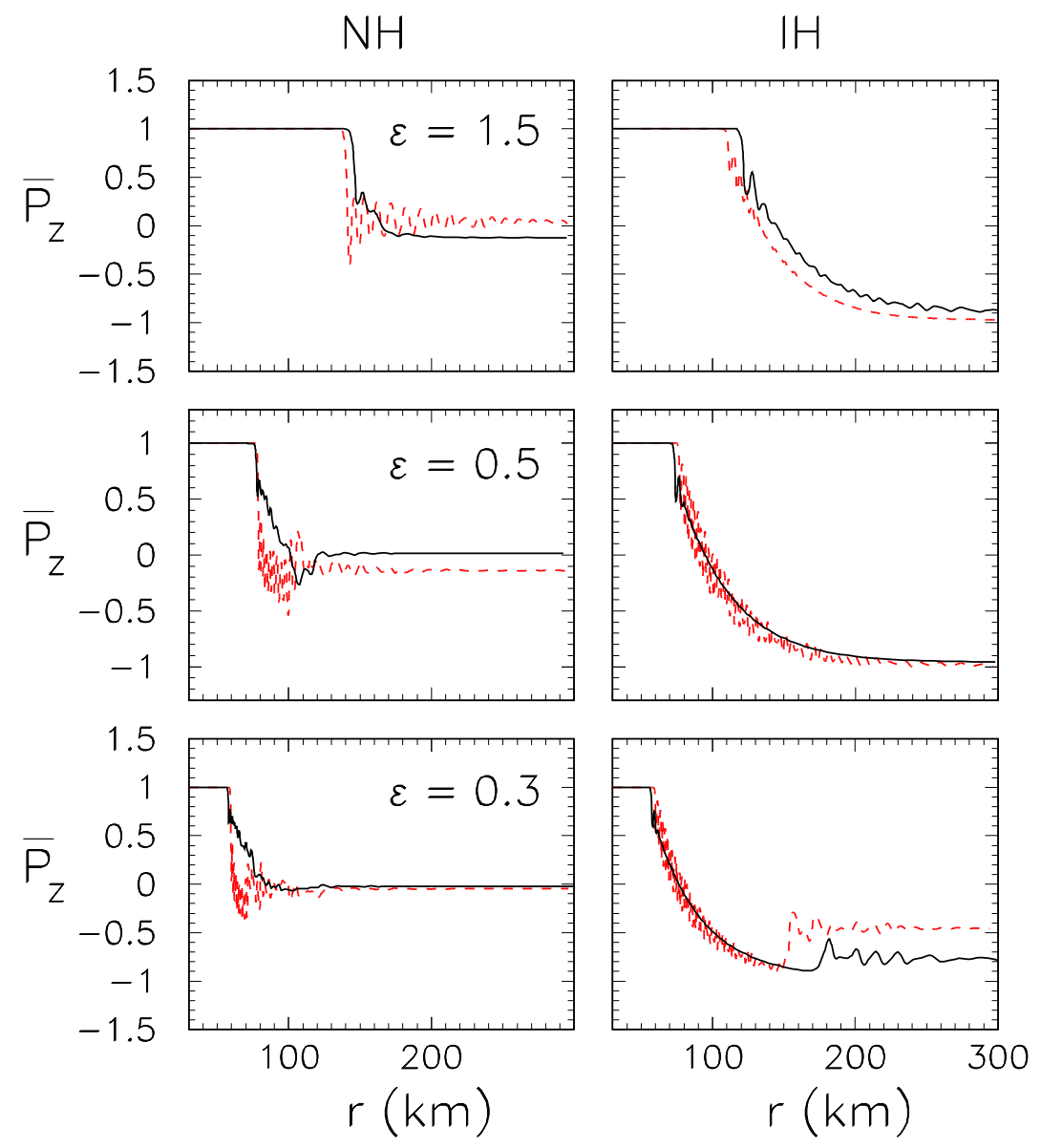

FIG. 1: Single energy case with $\omega_{0}=0.34 \mathrm{~km}^{-1}$. Radial evolution of the angle-integrated z-component $\bar{P}_{z}$ of the $\bar{\nu}$ polarization vector for different values of the flavor asymmetry $\varepsilon$. Left panels refer to normal hierarchy, right panels to inverted hierarchy. Dashed curves are for the MAA, SZA evolution; while continuous curves are for the MAA, MZA cases.

a certain fraction of $\nu_{e}$ in the final state. Passing now to $\mathrm{IH}$, we find that the onset of the flavor conversions is associated with the usual bimodal instability, leading to the well-known "pendulum" behaviour which tend to completely invert $\bar{P}_{z}$ leading to a complete $\bar{\nu}_{e} \rightarrow \bar{\nu}_{x}$ flavor change [17]. For $\varepsilon=1.5,0.5$, MAA and MZA effects do not play a significant role, since one observes a "quasi-single angle" behavior [33], driven by the bimodal instability. Only for a small flavor asymmetry $\varepsilon=0.3$, MAA effects can lead to a deviation with respect to the expected pendulum behavior. This can be understood as follows. We have found that an ensemble initially composed of only $\nu_{e}$ and $\bar{\nu}_{e}$ is unstable under MAA effects in $\mathrm{NH}$. Instead, if the initial ensemble had been consisted by $\nu_{x}$ and $\bar{\nu}_{x}$, the situation would have been reversed, i.e. we would have found the MAA instability for IH. Therefore, starting with $\nu_{e}$ and $\bar{\nu}_{e}$, the MAA instability can act in IH only after bimodal flavor conversions have significantly swapped the initial fluxes into the unstable $\nu_{x}$ and $\bar{\nu}_{x}$ ones. In our numerical example, only for $\varepsilon=0.3$, bimodal flavor conversions start early enough to allow the MAA instability to grow and significantly affect the flavor evolution at large $r$.

\section{FERMI-DIRAC NEUTRINO DISTRIBUTIONS}

It is known that self-induced flavor conversions can produce peculiar spectral features on continuous $\nu$ energy distributions, known as spectral swaps and splits [21]. At this regard, we find interesting to investigate how the new MAA instability acts on neutrino spectra. To explore this point, we take a system composed of only $\nu_{e}$ and $\bar{\nu}_{e}$ with an excess of $\nu_{e}$ parametrized in terms of the asymmetry parameter $\varepsilon$. This simple toy model is intended to roughly mimic the $\mathrm{SN} \nu$ emission, where one expects an excess of $\nu_{e}$ due to the core deleptonization.

We describe the $\nu$ spectra with Fermi-Dirac distributions with average energy $\langle E\rangle=15 \mathrm{MeV}$ and luminosity $L_{\bar{\nu}_{e}}=10^{51} \mathrm{erg} / \mathrm{s}$. In Fig. 2 we represent the initial $g(\omega)$ spectra for our three reference values of asymmetry parameter, namely $\varepsilon=1.5$ (upper panel), $\varepsilon=0.5$ (middle panel) and $\varepsilon=0.3$ (lower panel). These values are in the range of the asymmetries that one would expect during the different phases of the SN neutrino burst. 

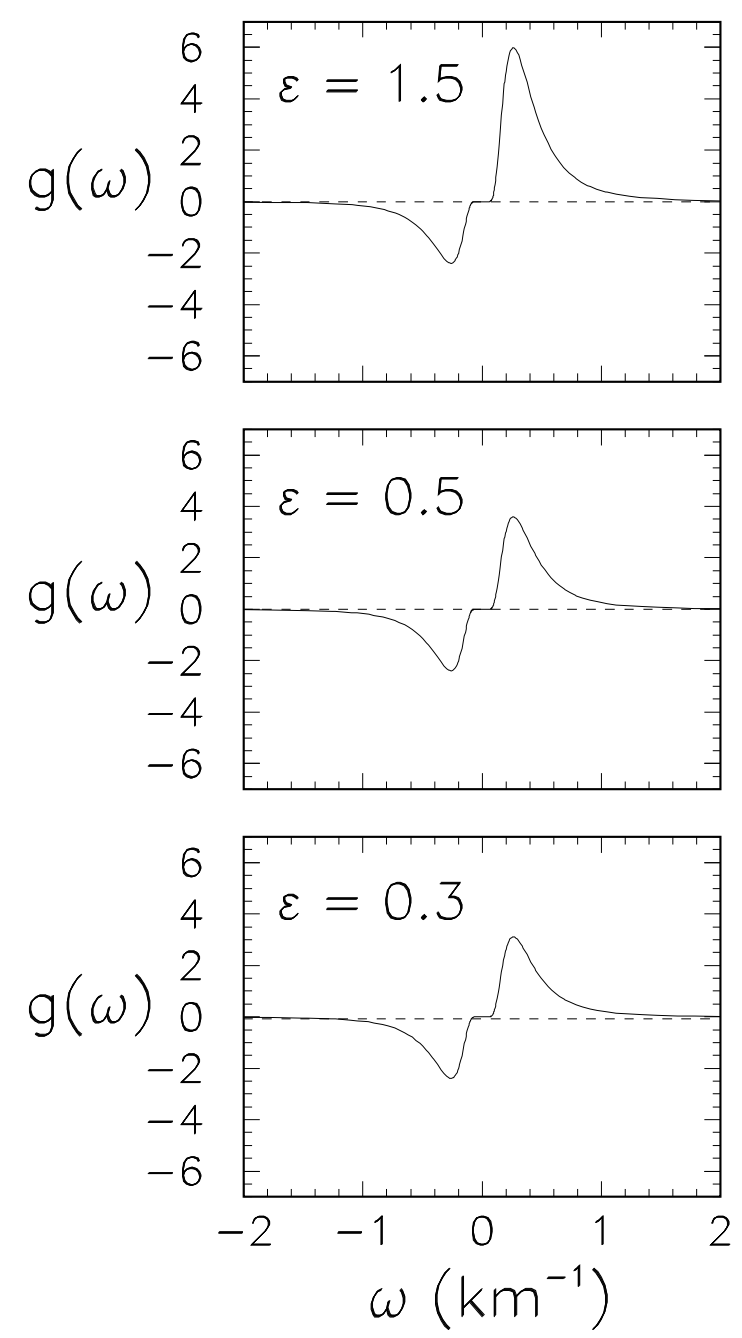

FIG. 2: $g(\omega)$ spectra corresponding to $\nu_{e}$ and $\bar{\nu}_{e}$ Fermi-Dirac distributions with $\langle E\rangle=15 \mathrm{MeV}$ and different values of the flavor asymmetry $\varepsilon$.

\section{A. Flavor evolution}

Starting with the spherically symmetric $g(\omega)$ at $R$ we evaluate the flavor evolution without axial symmetry in both $\mathrm{NH}$ and $\mathrm{IH}$. We use the same number of angular bins as in the previous Section. Moreover, we consider $N_{\omega}=80$ frequency modes corresponding to a range $E \in[0 ; 80] \mathrm{MeV}$. In Fig. 3 we represent the radial evolution of the $z$-component $\bar{P}_{z}$ of the $\bar{\nu}$ polarization vector $\mathbf{P}_{\omega, u, \varphi}$ integrated over $\omega, \varphi$ and $u$, in the same format of Fig. 1. We realize that the behavior of the integrated $\bar{P}_{z}$ is very similar to what we have seen in the single-energy example. In particular, new flavor conversions occur in normal hierarchy triggered by the MAA instability, with a final $\bar{P}_{z} \simeq 0$ for $\varepsilon=0.3,0.5$ and $\bar{P}_{z} \simeq-0.3$ for $\varepsilon=1.5$.

In order to have a better understanding of how modes with different energies evolve, in Fig. 4 and 5 we show the radial evolution of $P_{\omega, z}$ (integrated over both the angular variables) for two representative $\omega$ 's in NH (Fig. 4) and IH (Fig. [5) for the same values of $\varepsilon$ of Fig. [3 with MZA ef- fect. In particular, left panels refer to $\bar{\nu}$, while right panels to $\nu$. We have normalized the $\nu$ polarization vectors at the neutrinosphere as $P_{\omega, z}=\left(F_{e}^{R}-F_{x}^{R}\right) /\left(F_{e}^{R}+F_{x}^{R}\right)$ and analogously for $\bar{\nu}$. As energy modes, we show $\omega=0.49 \mathrm{~km}^{-1}$ (dot-dashed curves) and $\omega=0.29 \mathrm{~km}^{-1}$ (dotted curves) We start discussing the NH in Fig. 4. For large value of $\varepsilon=1.5$ (upper panels) we realize that, the $\bar{P}_{\omega, z}$ for the two different frequency modes tend to invert with respect to the initial value. Conversely in the $\nu$ sector (right panels) the $\omega=0.29 \mathrm{~km}^{-1}$ mode do invert its $P_{\omega, z}$ with respect to their initial value, while the $\omega=0.49 \mathrm{~km}^{-1}$ mode returns to its original value at the end of the flavor evolution. Therefore, the two different energy modes exhibit a behavior that presumably suggests the occurrence of splitting features in the final spectra, with some parts of the spectra equal to the original ones and other partially or completely swapped to the other flavor. Lowering the value of the asymmetry parameter $\varepsilon$ this behavior seems to change. In particular for $\varepsilon=0.3,0.5, \bar{P}_{\omega, z}$ tend to saturate at 0 for the two 

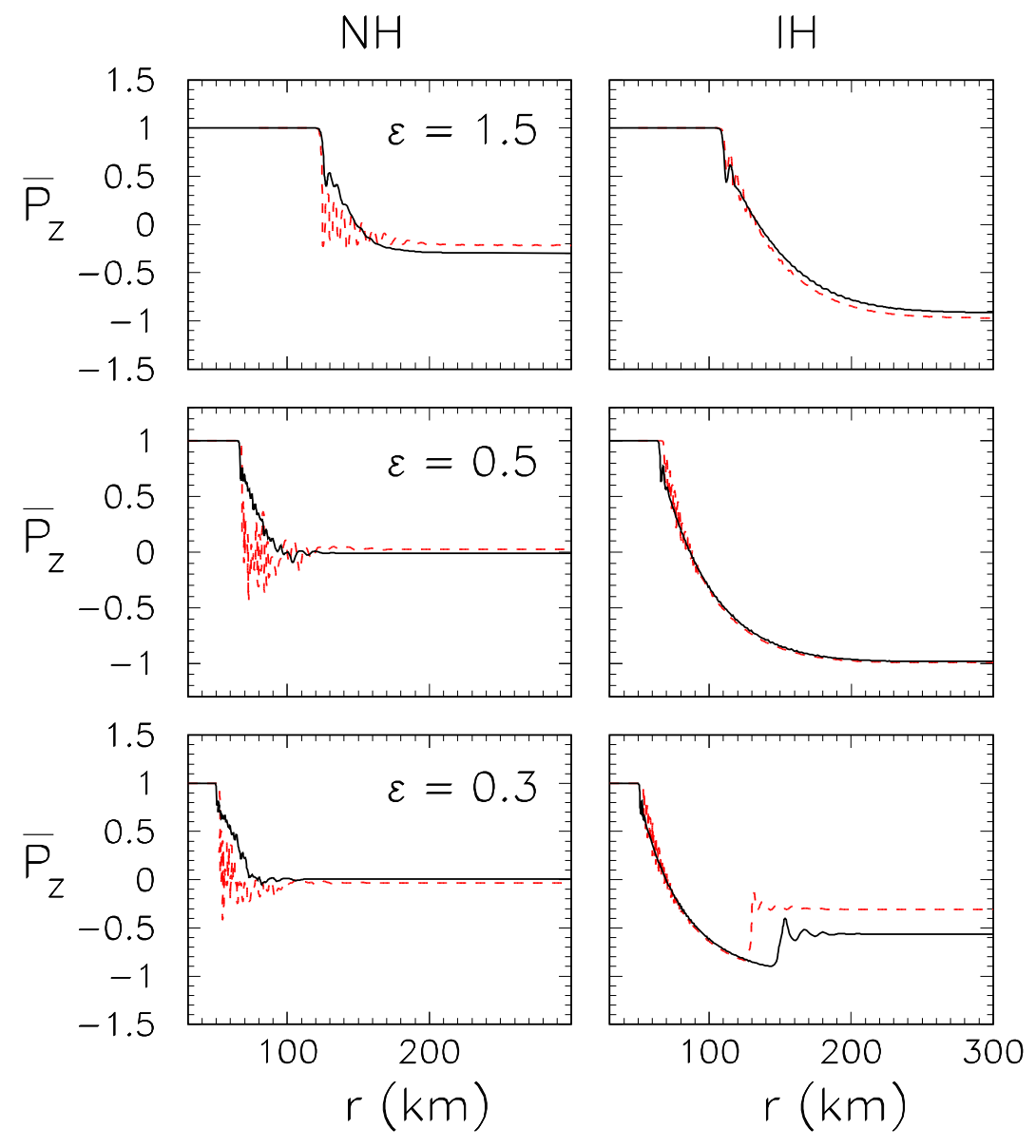

FIG. 3: Multi-energy case corresponding to Fermi-Dirac energy spectra with $\langle E\rangle=15 \mathrm{MeV}$. Radial evolution of the integrated z-component $\bar{P}_{z}$ of the polarization vector for $\bar{\nu}$ for different values of the flavor asymmetry $\varepsilon$. Left panels refer to normal hierarchy, right panels to inverted hierarchy. Dashed curves are for the MAA, SZA evolution; while continuous curves are for the MAA, MZA case.

different energy modes. In the $\nu$ sector the final $P_{\omega, z}$ maintain a finite final value as imposed by the conservation of the total asymmetry $\varepsilon$. In our example, it seems that a larg value $\varepsilon \sim 1$ is required to prevent flavor equilibrium induced by MAA effects.

Passing now to the IH case of Fig. 5 , for $\varepsilon=1.5,0.5$ we find the known evolution due to the bimodal instability [19, 20]. In particular, the $\bar{\nu}$ modes invert $\bar{P}_{\omega, z}$ with respect to the initial value, while for the $\nu, P_{\omega, z}$ comes back to the initial value for $\omega=0.49 \mathrm{~km}^{-1}$ mode and invert their value for the other lower frequency mode. This behavior would produce a spectral split in the $\nu$ sector and an almost complete swap in the $\bar{\nu}$ channel. Finally, if we consider the case with $\varepsilon=0.3$ we realize that MAA instability produces a modification in the flavor evolution around $r \simeq 140 \mathrm{~km}$, disturbing the expected dynamics associated with the bimodal evolution. As a consequence one can foresee a smearing of the splitting features produced for larger $\varepsilon$.

\section{B. Oscillated spectra}

In order to show how this flavor dynamics triggered by the MAA effects impacts the oscillated $\nu$ spectra, in the following three Figures we represent in the upper panels the initial $g(\omega)$ (light continuous curves), the final one under MAA flavor evolution with SZA effect (dashed curve) and the final one with MZA effect (thick continuous curves). In the lower panels we represent the swap function $s(\omega)=g^{\text {final }}(\omega) / g^{\text {initial }}(\omega)$ for the SZA evolution (dashed curves) and the MZA case (thick continuous curves). Left panels refer to $\mathrm{NH}$, while right panels to IH. In particular, Fig. 6 is for $\varepsilon=1.5$, Fig. 7 for $\varepsilon=0.5$ and Fig. 8 for $\varepsilon=0.3$.

Starting with $\varepsilon=1.5$ in Fig. 6, we realize that the final spectra in $\mathrm{NH}$ and $\mathrm{IH}$ are qualitatively similar. The MAA instability in $\mathrm{NH}$ produces a final spectrum with a split at $\omega \simeq 0.2 \mathrm{~km}^{-1}$, similar to the one induced in IH by the bimodal instability at $\omega \simeq 0.4 \mathrm{~km}^{-1}$. In the SZA evolution another split would appear in the $\bar{\nu}$ at $\omega \simeq-0.5 \mathrm{~km}^{-1}$ in $\mathrm{NH}$, and at $\omega \simeq-1.5 \mathrm{~km}^{-1}$ in IH. 

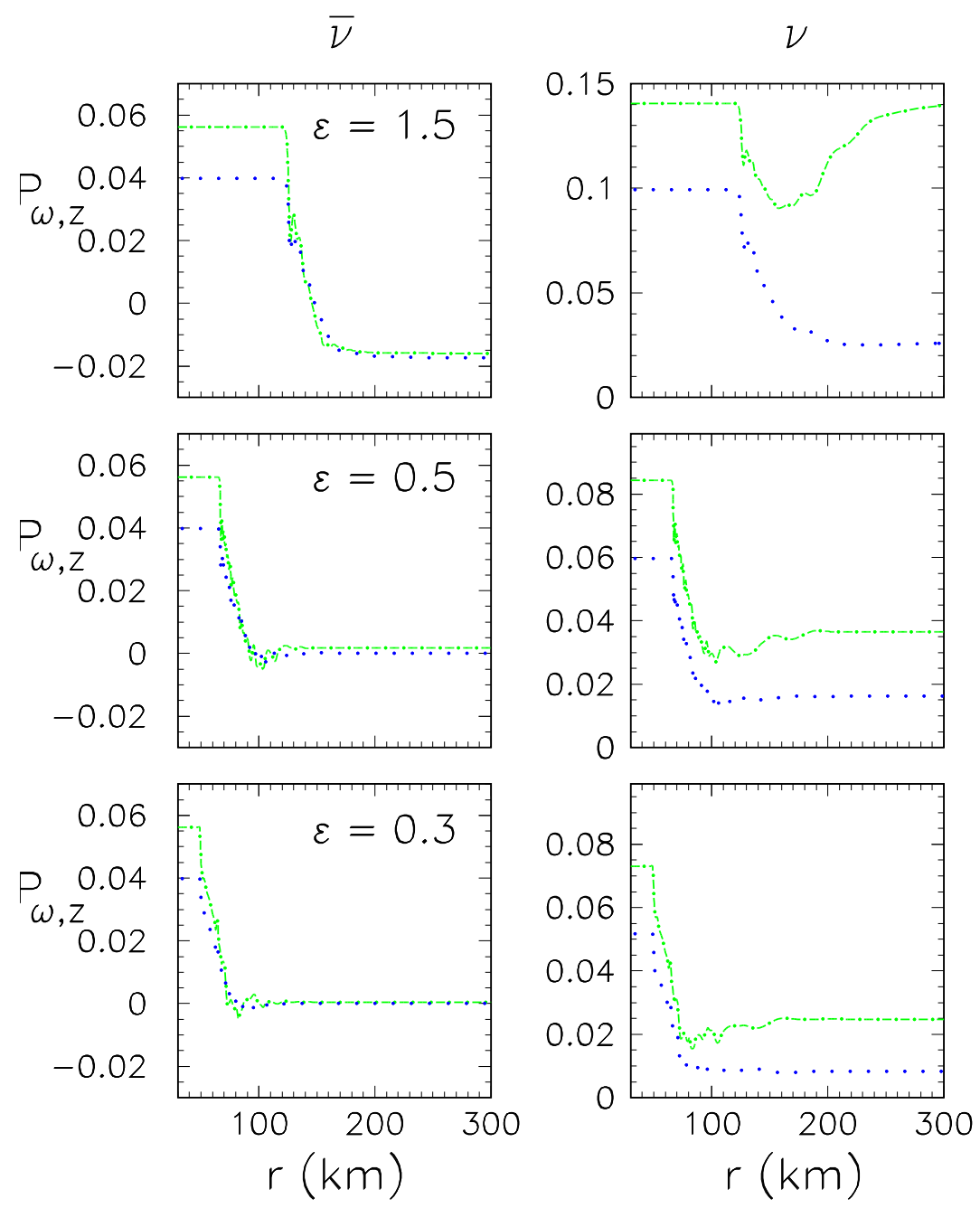

FIG. 4: Normal hierarchy. Evolution of two different $\omega$ modes $P_{\omega, z}$ for $\bar{\nu}_{e}$ (left panels) and $\nu_{e}$ (right panels) for different values of the flavor asymmetry $\varepsilon$ in MAA, MZA case of Fig. 3 The two lines correspond $\omega=0.49 \mathrm{~km}^{-1}$ (dot-dashed curves) and $\omega=0.29 \mathrm{~km}^{-1}$ (dotted curves), respectively.

However, both these $\bar{\nu}$ splits are rather fragile under the MZA effects, which tend to smear them.

In Fig. 7 we consider $\varepsilon=0.5$. We realize that IH evolution is very similar to the one observed for $\varepsilon=1.5$, apart from the disappearance of the $\bar{\nu}$ split. Conversely, in NH the final spectrum is remarkably different. From the swap function $s(\omega)$ one observes that the swaps and splits are significantly smeared in both SZA and MZA situations. The swap function for $\bar{\nu}$ tends to flatten, approaching zero in the $\bar{\nu}$ sector, indicating the tendency towards flavor decoherence.

Finally in Fig. 8 we consider $\varepsilon=0.3$. In $\mathrm{NH}$ the flavor decoherence is complete with $s(\omega) \sim 0$ for $\bar{\nu}$. For $\nu$ for $\omega>0.5 \mathrm{~km}^{-1}$ one finds $s(\omega) \sim 0$ in SZA, while $s(\omega) \sim 0.5$ in MZA. For smaller $\omega$ the swap function tends towards zero in both the situations. In IH, the MAA effects produce a strong smearing of the splitting features. Multiple splits appear along the spectrum. In the $\bar{\nu}$ part the SZA and MZA cases exhibit a different behavior for $\omega<-0.4 \mathrm{~km}^{-1}$. In particular, $s(\omega) \simeq 0$ in the SZA scheme, while $s(\omega) \simeq-1$ in the MZA evolution.

From these results, one can conclude that the new found MAA instability can lead to a rich phenomenology. The general outcome of the flavor evolution is not a trivial flavor decoherence as one could have expected from the single-energy evolution. Conversely, an ordered behavior can be present in the final spectra. As for the MZA effect, a crucial parameter to suppress the decoherence is the flavor asymmetry parameter $\varepsilon$. However, at least from our numerical examples, it seems that a large value of $\varepsilon$ (i.e. $\varepsilon \sim 1$ ) is required to suppress the MAA decoherence, while it was found that a smaller value, i.e. $\varepsilon \gtrsim 0.3$, is enough to suppress the MZA instability [33. 


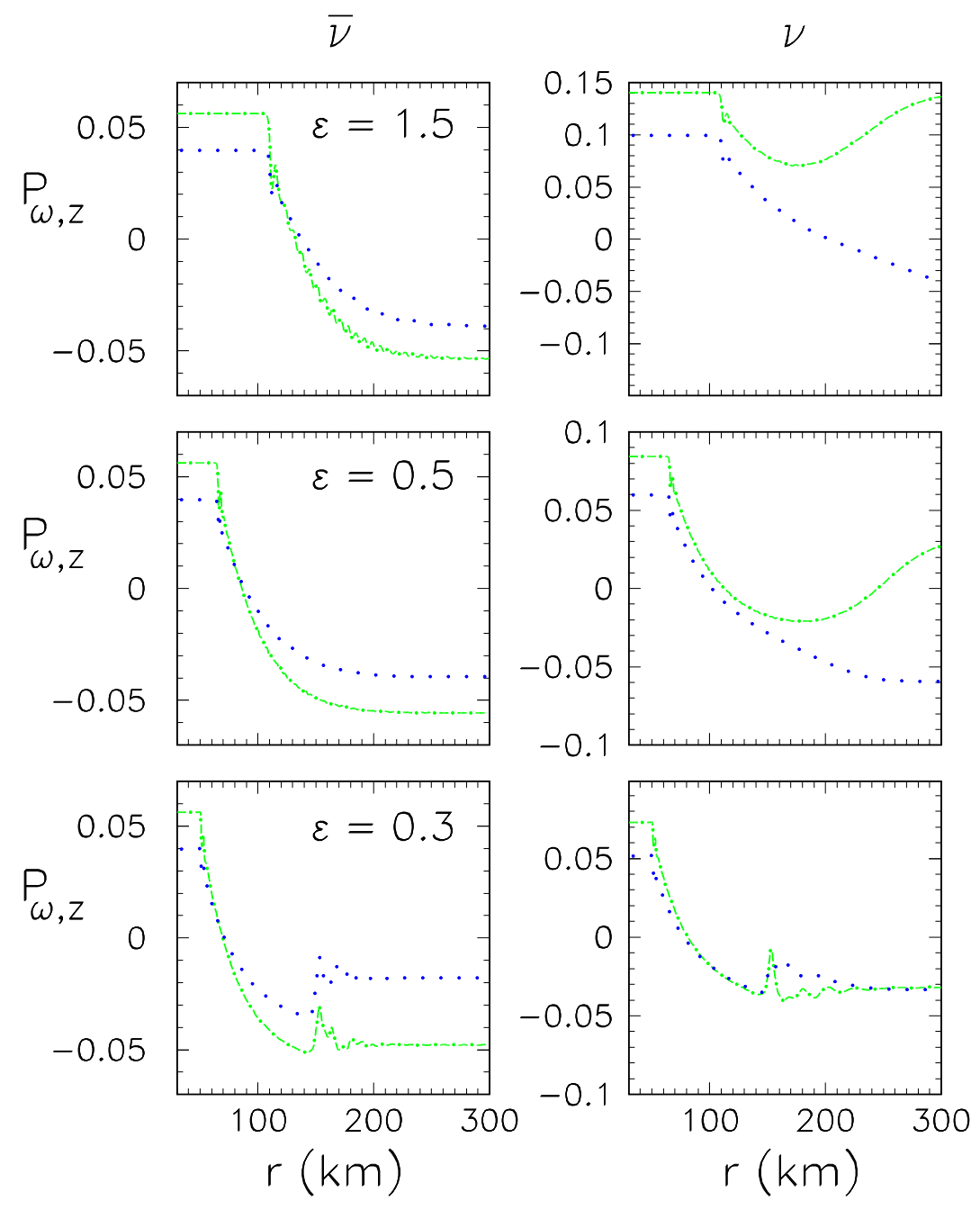

FIG. 5: Inverted hierarchy. Evolution of two different $\omega$ modes $P_{\omega, z}$ for $\bar{\nu}_{e}$ (left panels) and $\nu_{e}$ (right panels) for different values of the flavor asymmetry $\varepsilon$ in MAA, MZA case of Fig. 3 The two lines correspond $\omega=0.49 \mathrm{~km}^{-1}$ (dot-dashed curves) and $\omega=0.29 \mathrm{~km}^{-1}$ (dotted curves), respectively.

\section{Multipoles evolution}

When the axial symmetry is broken in the flavor evolution, the polarization vectors can develop significant multipoles in $\cos \varphi$ or $\sin \varphi$. At this regard, in order to compare the development of MAA effects in the different cases we studied, we find interesting to show the development of the multipole expansion of the polarization vectors in $\cos \varphi$.

Since the onset of the MAA instability is related to an exponential growth of the transverse components $(x, y)$ of the polarization vector, we define

$$
P_{m}^{x, y}=\frac{1}{2 \pi} \int d \Gamma g(\omega) P_{\omega, u, \varphi}^{x, y} \cos m \varphi
$$

and

$$
P_{m}=\sqrt{\left(P_{m}^{x}\right)^{2}+\left(P_{m}^{y}\right)^{2}},
$$

where $m=1,2,3 \ldots$ In Fig. 9 we represent the first two multipoles $P_{m}$ for $\bar{\nu}$ for the MAA evolution in the MZA case shown in Fig. 3 using the same format of that Figure. In particular, the continuous curves refer to the dipole $m=1$, while the dashed one to $m=2$. Starting from $\varepsilon=1.5$ we see that in $\mathrm{NH} P_{1}$ rises exponentially to $\sim 10^{-2}$ at $r \simeq 100 \mathrm{~km}$ corresponding to the onset of the flavor conversions. Then, it declines by roughly one order of magnitude at $r \simeq 200 \mathrm{~km}$. It is interesting to realize that the $m=2$ multipole at its peak is suppressed by roughly two orders of magnitude with respect to $P_{1}$. In $\mathrm{IH}$, no multipole develops since the polarization vectors maintain a spherically symmetric distribution. Passing now to the case of $\varepsilon=0.5$ we realize that the $P_{m}$ rises at $r \simeq 70 \mathrm{~km}$. In comparison with the previous case we see that $P_{2}$ at the peak is only a factor $\sim 3$ smaller than $P_{1}$. Moreover, at $r \gtrsim 100 \mathrm{~km}$ the two different multipoles have a comparable strength. This seems an important difference with respect to the $\varepsilon=1.5$ case and 

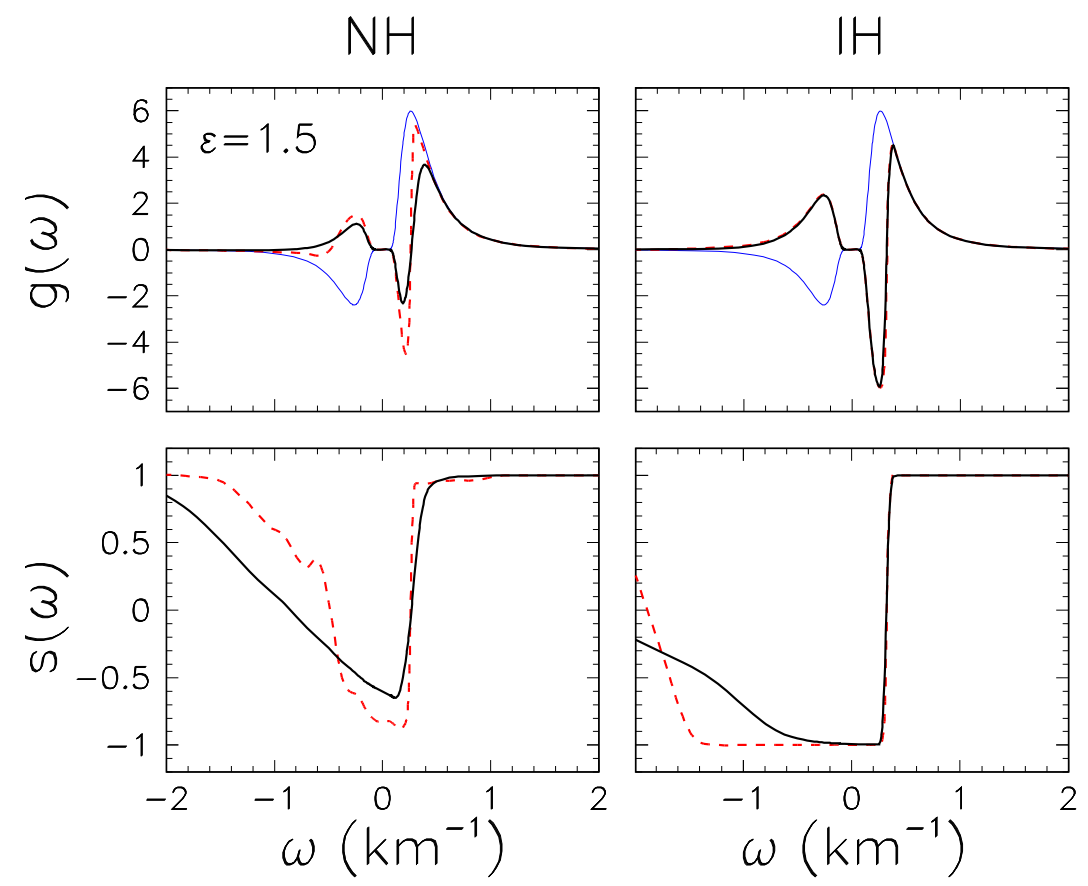

FIG. 6: Asymmetry parameter $\varepsilon=1.5$. Upper panels: initial (thin continuous curves) and final $\nu$ spectrum for the MAA evolution in SZA (dashed curves) and MZA (thick continuous curves) case for normal hierarchy (left panel) and inverted hierarchy (right panel). Lower panels: swap function, i.e. ratio of final with initial spectra in SZA (dashed curves) and MZA (thick continuous curves).
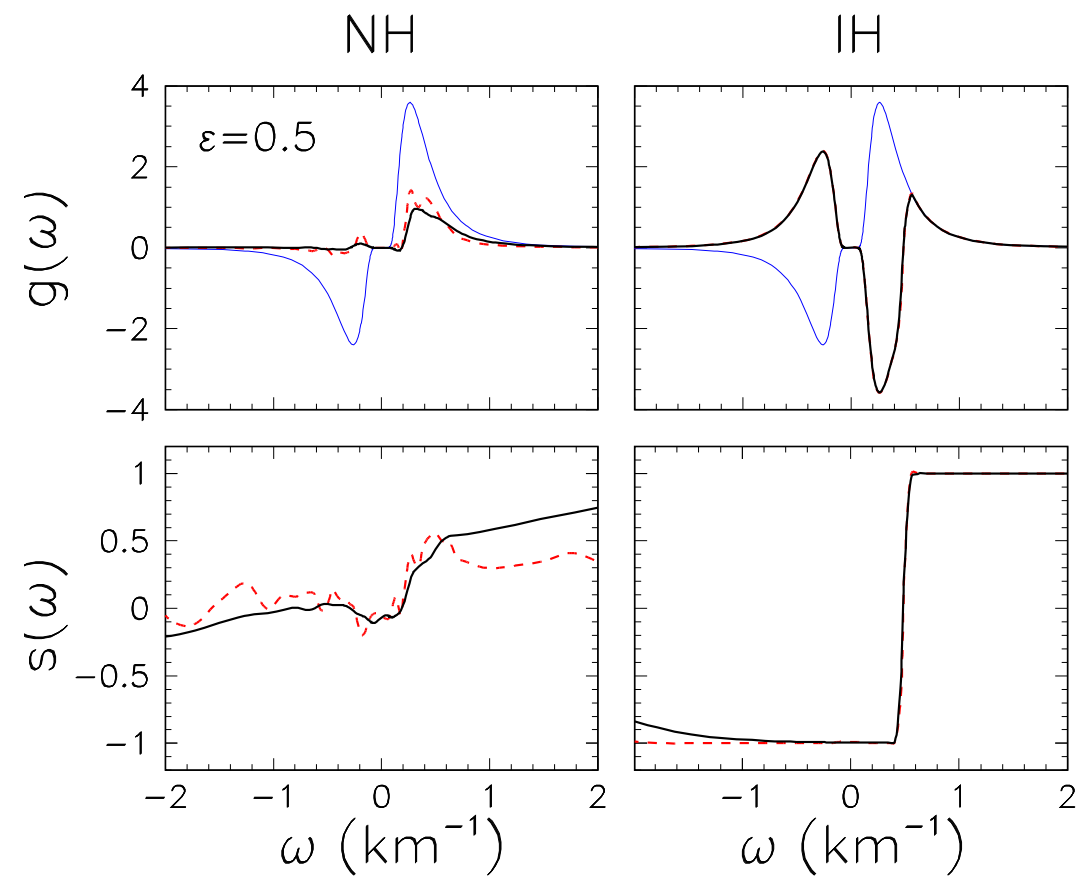

FIG. 7: Asymmetry parameter $\varepsilon=0.5$. Upper panels: initial (thin continuous curves) and final $\nu$ spectrum for the MAA evolution in SZA (dashed curves) and MZA (thick continuous curves) case for normal hierarchy (left panel) and inverted hierarchy (right panel). Lower panels: swap function, i.e. ratio of final with initial spectra in SZA (dashed curves) and MZA (thick continuous curves).

corresponds to a different flavor evolution. In particular, for $\varepsilon=1.5$ where a rather ordered behavior in the polarization vector occurs, only the dipole is significantly excited. Conversely, for $\varepsilon=0.5$ where the $\nu$ system tends 

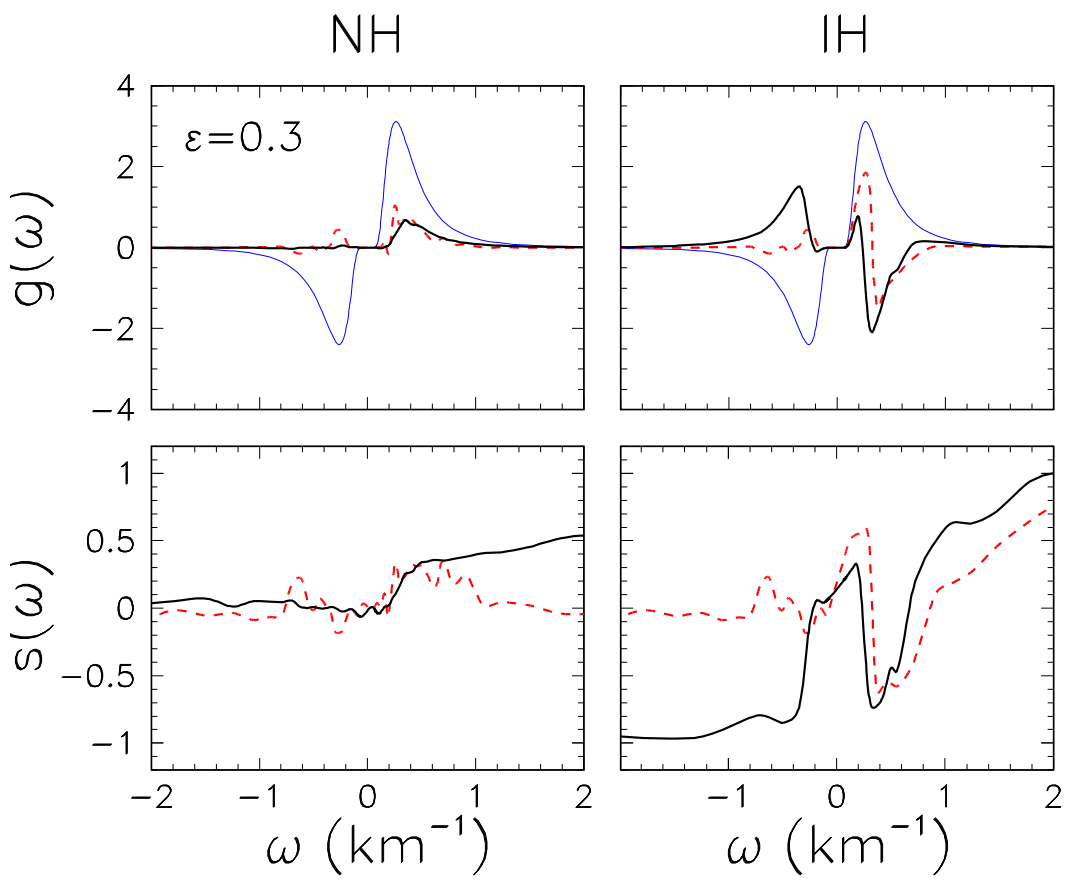

FIG. 8: Asymmetry parameter $\varepsilon=0.3$. Upper panels: initial (thin continuous curves) and final $\nu$ spectrum for the MAA evolution in SZA (dashed curves) and MZA (thick continuous curves) case for normal hierarchy (left panel) and inverted hierarchy (right panel). Lower panels: swap function, i.e. ratio of final with initial spectra in SZA (dashed curves) and MZA (thick continuous curves).

to evolve towards the flavor decoherence, also higher order multipoles are significantly excited. In this case in $\mathrm{IH}$ again one does not observe any development of multipoles.

Finally, for $\varepsilon=0.3$ the $\mathrm{NH}$ has a behavior similar to the previous one, with the different multipoles significantly excited at $r \simeq 50 \mathrm{~km}$. Moreover, in IH the different multipoles get excited at $r \simeq 140 \mathrm{~km}$, where the MAA instability starts to develop after the bimodal instability swapped the original spectra.

\section{CONCLUSIONS}

Very recently it has been pointed out that removing the axial symmetry in the solution of the non-linear evolution equations of SN neutrinos, a new multi-azimuthal-angle instability would emerge [1, 38]. Inspired by this important finding, we performed the first simulations of the SN neutrino flavor evolution including the azimuthal angle $\varphi$ as explicit variable. In order to understand this flavor dynamics, we considered simple toy models in which we prepared our system initially with an excess of $\nu_{e}$ over $\bar{\nu}_{e}$. We started considering monochromatic neutrino beams. In this case, in NH significant flavor conversions are triggered by the MAA instability generically leading to flavor decoherence of the neutrino ensemble. In IH the MAA instability can affect the flavor evolution only for small neutrino asymmetries, but only after the usual bimodal instability has inverted the initial flavor content. Con- sidering more realistic continuous energy distributions, described by Fermi-Dirac energy spectra, we find that the MAA instability can lead to a rich phenomenology. Indeed, the flavor decoherence found in the single-energy case is not the unique possibility. In particular, for large flavor asymmetries, oscillated spectra exhibit an ordered behavior with swaps and splits, similar to the ones produces in IH by the bimodal instability. Only for small flavor asymmetries the flavor decoherence is unavoidable.

This result observed with our simple examples would presumably lead to a change of paradigm in the characterization of self-induced SN neutrino oscillations. In order to assess the impact of the MMA instability on the flavor evolution of SN neutrinos, one necessarily would move from toy models to a more realistic characterization of the dense SN neutrino gas. In particular, it is well-known that the self-induced effects are crucially dependent on the flux ordering among different neutrino species (see, e.g., [30]). Moreover, the role of dense ordinary matter, that could suppress the self-induced effects during the accretion phase [39 42] needs to be explored. Indeed, according to the stability analysis performed in 1], in the presence of MAA effects matter suppression would require larger densities than previously thought. Furthermore, in the present study we have assumed perfect spherical neutrino emission, which does not occur in multi-dimensional SN simulations [49, 50]. In future works it would be interesting to understand how the removal of the initial spherical symmetry would affect the further flavor evolution. 

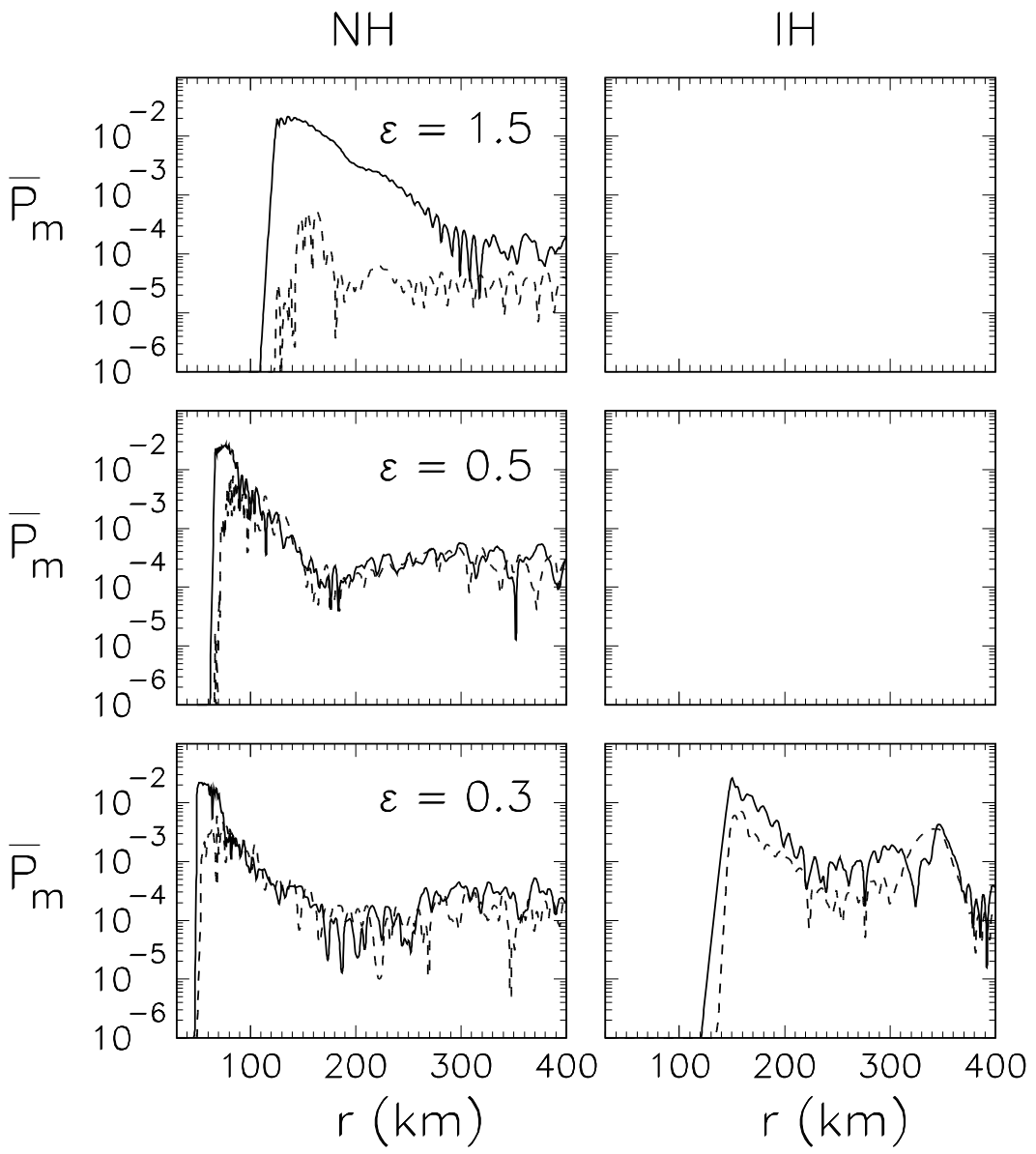

FIG. 9: Radial evolution of the multipoles $P_{m}$ for $\bar{\nu}$ in the MAA evolution for the MZA case of Fig. 3 . Left panels refer to normal hierarchy, right panels to inverted hierarchy. Continuous curves refer to $m=1$, dashed ones to $m=2$.

In conclusion, the discovery of these new effects adds many open questions and additional layers of complications in the simulation of the flavor evolution for supernova neutrinos. At the moment previous results of the self-induced effects on SN neutrinos, based on the unjustified assumption of axial symmetry could not be taken as granted, but should be reexamined. For sure, further studies are necessary to characterize this fascinating flavor dynamics and to get a deeper theoretical understanding of its effects. At this regard, the possibility to detect signatures of self-induced oscillations in the next galactic supernova neutrino burst [48] would motivate further analytical and numerical investigations.

\section{Acknowledgements}

A.M. kindly thanks Georg Raffelt for many interesting discussions and suggestions during the development of this work. Moreover, he acknowledges Basudeb Dasgupta, Huaiyu Duan and Irene Tamborra for interesting comments on the manuscript. This work was supported by the German Science Foundation (DFG) within the Collaborative Research Center 676 "Particles, Strings and the Early Universe."
[1] G. Raffelt, S. Sarikas and D. d. S. Seixas, Phys. Rev. Lett. 111, 091101 (2013) arXiv:1305.7140 [hep-ph]].

[2] L. Wolfenstein, "Neutrino Oscillations In Matter," Phys. Rev. D 17, 2369 (1978); S. P. Mikheev and A. Yu. Smirnov, "Resonance Enhancement Of Oscillations In Matter And Solar Neutrino Spectroscopy," Yad. Fiz. 42, 1441 (1985) [Sov. J. Nucl. Phys. 42, 913 (1985)].

[3] A. S. Dighe and A. Y. Smirnov, "Identifying the neutrino mass spectrum from the neutrino burst from a supernova," Phys. Rev. D 62, 033007 (2000) hep-ph/9907423.

[4] J. Pantaleone, "Neutrino oscillations at high densities," Phys. Lett. B 287, 128 (1992).

[5] G. Sigl and G. Raffelt, "General kinetic description of relativistic mixed neutrinos," Nucl. Phys. B 406, 423 (1993).

[6] B. H. J. McKellar and M. J. Thomson, "Oscillating dou- 
blet neutrinos in the early universe," Phys. Rev. D 49 , 2710 (1994).

[7] Y. Z. Qian and G. M. Fuller, "Neutrino-neutrino scattering and matter enhanced neutrino flavor transformation in Supernovae," Phys. Rev. D 51, 1479 (1995) astro-ph/9406073.

[8] S. Samuel, "Neutrino oscillations in dense neutrino gases," Phys. Rev. D 48, 1462 (1993).

[9] V. A. Kostelecký and S. Samuel, "Neutrino oscillations in the early universe with an inverted neutrino mass hierarchy," Phys. Lett. B 318, 127 (1993).

[10] V. A. Kostelecký and S. Samuel, "Self-maintained coherent oscillations in dense neutrino gases," Phys. Rev. D 52, 621 (1995) hep-ph/9506262.

[11] S. Samuel, "Bimodal coherence in dense selfinteracting neutrino gases," Phys. Rev. D 53, 5382 (1996) hep-ph/9604341.

[12] S. Pastor, G. G. Raffelt and D. V. Semikoz, "Physics of synchronized neutrino oscillations caused by self-interactions," Phys. Rev. D 65, 053011 (2002) hep-ph/0109035.

[13] S. Pastor and G. Raffelt, "Flavor oscillations in the supernova hot bubble region: Nonlinear effects of neutrino background," Phys. Rev. Lett. 89, 191101 (2002) astro-ph/0207281.

[14] R. F. Sawyer, "Speed-up of neutrino transformations in a supernova environment," Phys. Rev. D 72, 045003 (2005) hep-ph/0503013.

[15] H. Duan, G. M. Fuller and Y. Z. Qian, "Collective Neutrino Flavor Transformation In Supernovae," Phys. Rev. D 74, 123004 (2006) astro-ph/0511275.

[16] H. Duan, G. M. Fuller, J. Carlson and Y. Z. Qian, "Simulation of coherent non-linear neutrino flavor transformation in the supernova environment. I: Correlated neutrino trajectories," Phys. Rev. D 74, 105014 (2006) astro-ph/0606616.

[17] S. Hannestad, G. G. Raffelt, G. Sigl and Y. Y. Y. Wong, "Self-induced conversion in dense neutrino gases: Pendulum in flavour space," Phys. Rev. D 74, 105010 (2006) [Erratum-ibid. D 76, 029901 (2007)] astro-ph/0608695].

[18] H. Duan, G. M. Fuller and Y. -Z. Qian, "Collective Neutrino Oscillations," Ann. Rev. Nucl. Part. Sci. 60, 569 (2010) arXiv:1001.2799 [hep-ph]].

[19] G. L. Fogli, E. Lisi, A. Marrone and A. Mirizzi, "Collective neutrino flavor transitions in supernovae and the role of trajectory averaging," JCAP 0712, 010 (2007) arXiv:0707.1998 [hep-ph]].

[20] G. L. Fogli, E. Lisi, A. Marrone, A. Mirizzi and I. Tamborra, "Low-energy spectral features of supernova (anti)neutrinos in inverted hierarchy," Phys. Rev. D 78, 097301 (2008) arXiv:0808.0807 [hep-ph]].

[21] G. G. Raffelt and A. Y. Smirnov, "Self-induced spectral splits in supernova neutrino fluxes," Phys. Rev. D 76, 081301 (2007) [Erratum-ibid. D 77, 029903 (2008)] arXiv:0705.1830 [hep-ph]].

[22] G. G. Raffelt, A. Y. Smirnov, "Adiabaticity and spectral splits in collective neutrino transformations," Phys. Rev. D76, 125008 (2007). arXiv:0709.4641 [hep-ph]].

[23] H. Duan, G. M. Fuller, J. Carlson et al., "Neutrino Mass Hierarchy and Stepwise Spectral Swapping of Supernova Neutrino Flavors," Phys. Rev. Lett. 99, 241802 (2007). arXiv:0707.0290 [astro-ph]].

[24] H. Duan, G. M. Fuller, Y. -Z. Qian, "Stepwise spectral swapping with three neutrino flavors," Phys. Rev. D77,
085016 (2008). arXiv:0801.1363 [hep-ph]].

[25] J. Gava and C. Volpe, "Collective neutrinos oscillation in matter and CP-violation," Phys. Rev. D 78, 083007 (2008) arXiv:0807.3418 [astro-ph]].

[26] J. Gava, J. Kneller, C. Volpe and G. C. McLaughlin, "A dynamical collective calculation of supernova neutrino signals," Phys. Rev. Lett. 103, 071101 (2009) arXiv:0902.0317 [hep-ph]].

[27] B. Dasgupta, A. Dighe, G. G. Raffelt and A. Y. Smirnov, "Multiple Spectral Splits of Supernova Neutrinos," Phys. Rev. Lett. 103, 051105 (2009) arXiv:0904.3542 [hep$\mathrm{ph}]]$.

[28] A. Friedland, "Self-refraction of supernova neutrinos: mixed spectra and three-flavor instabilities," Phys. Rev. Lett. 104, 191102 (2010) arXiv:1001.0996 [hep-ph]].

[29] B. Dasgupta, A. Mirizzi, I. Tamborra and R. Tomàs, "Neutrino mass hierarchy and three-flavor spectral splits of supernova neutrinos," Phys. Rev. D 81, 093008 (2010) arXiv:1002.2943 [hep-ph]].

[30] A. Mirizzi and R. Tomàs, "Multi-angle effects in self-induced oscillations for different supernova neutrino fluxes," Phys. Rev. D 84, 033013 (2011) arXiv:1012.1339 [hep-ph]].

[31] J. T. Pantaleone, "Dirac neutrinos in dense matter," Phys. Rev. D 46, 510 (1992).

[32] G. G. Raffelt and G. Sigl, "Self-induced decoherence in dense neutrino gases," Phys. Rev. D 75, 083002 (2007) hep-ph/0701182.

[33] A. Esteban-Pretel, S. Pastor, R. Tomàs, G. G. Raffelt and G. Sigl, "Decoherence in supernova neutrino transformations suppressed by deleptonization," Phys. Rev. D 76, 125018 (2007) arXiv:0706.2498 [astro-ph]].

[34] R. F. Sawyer, "The multi-angle instability in dense neutrino systems," Phys. Rev. D 79 (2009) 105003 arXiv:0803.4319 [astro-ph]].

[35] A. Mirizzi and P. D. Serpico, "Instability in the Dense Supernova Neutrino Gas with Flavor-Dependent Angular Distributions," Phys. Rev. Lett. 108, 231102 (2012) arXiv:1110.0022 [hep-ph]].

[36] A. Mirizzi and P. D. Serpico, "Flavor Stability Analysis of Dense Supernova Neutrinos with Flavor-Dependent Angular Distributions," Phys. Rev. D 86, 085010 (2012) arXiv:1208.0157 [hep-ph]].

[37] A. Banerjee, A. Dighe and G. Raffelt, "Linearized flavorstability analysis of dense neutrino streams," Phys. Rev. D 84, 053013 (2011) arXiv:1107.2308 [hep-ph]]. [38]

[38] G. Raffelt and D. d. S. Seixas, Phys. Rev. D 88, 045031 (2013) arXiv:1307.7625 [hep-ph]].

[39] S. Chakraborty, T. Fischer, A. Mirizzi, N. Saviano and R. Tomas, "No collective neutrino flavor conversions during the supernova accretion phase," Phys. Rev. Lett. 107, 151101 (2011) arXiv:1104.4031 [hep-ph]].

[40] S. Chakraborty, T. Fischer, A. Mirizzi, N. Saviano and R. Tomas, "Analysis of matter suppression in collective neutrino oscillations during the supernova accretion phase," Phys. Rev. D 84, 025002 (2011) arXiv:1105.1130 [hep-ph]].

[41] N. Saviano, S. Chakraborty, T. Fischer and A. Mirizzi, "Stability analysis of collective neutrino oscillations in the supernova accretion phase with realistic energy and angle distributions," Phys. Rev. D 85, 113002 (2012) arXiv:1203.1484 [hep-ph]].

[42] S. Sarikas, G. G. Raffelt, L. Hudepohl and H. -T. Janka, 
"Suppression of Self-Induced Flavor Conversion in the Supernova Accretion Phase," Phys. Rev. Lett. 108, 061101 (2012) arXiv:1109.3601 [astro-ph.SR]].

[43] J. F. Cherry, J. Carlson, A. Friedland, G. M. Fuller and A. Vlasenko, "Neutrino scattering and flavor transformation in supernovae," Phys. Rev. Lett. 108, 261104 (2012) arXiv:1203.1607 [hep-ph]].

[44] S. Sarikas, I. Tamborra, G. Raffelt, L. Hudepohl and H. -T. Janka, "Supernova neutrino halo and the suppression of self-induced flavor conversion," Phys. Rev. D 85, 113007 (2012) arXiv:1204.0971 [hep-ph]].

[45] http://www.nag.com/numeric/fl/manual/html /FLlibrarymanual.asp

[46] S. Sarikas, D. d. S. Seixas and G. Raffelt, "Spurious instabilities in multi-angle simulations of collective flavor conversion," Phys. Rev. D 86, 125020 (2012) arXiv:1210.4557 [hep-ph]].

[47] B. Dasgupta, A. Mirizzi, I. Tamborra and R. Tomas,
"Neutrino mass hierarchy and three-flavor spectral splits of supernova neutrinos," Phys. Rev. D 81 (2010) 093008 arXiv:1002.2943 [hep-ph]].

[48] S. Choubey, B. Dasgupta, A. Dighe and A. Mirizzi, "Signatures of collective and matter effects on supernova neutrinos at large detectors," arXiv:1008.0308 [hep-ph].

[49] C. D. Ott, A. Burrows, L. Dessart and E. Livne, "2D Multi-Angle, Multi-Group Neutrino RadiationHydrodynamic Simulations of Postbounce Supernova Cores," Astrophys. J. 685, 1069 (2008) arXiv:0804.0239 [astro-ph]].

[50] I. Tamborra, F. Hanke, B. Mueller, H. -T. Janka and G. Raffelt, "Neutrino signature of supernova hydrodynamical instabilities in three dimensions," Phys. Rev. Lett. 111, 121104 (2013) arXiv:1307.7936 [astroph.SR]]. 